\title{
Manufacturing challenges in the production of high quality modified-release tablets
}

\author{
Denis Ring
}

School of Engineering, UCC

Knowledge is the only instrument of production that is not subject to diminishing returns (John Maurice Clarke)

\section{Introduction}

The pharmaceutical industry is obliged and regulated to ensure that manufactured medicines (i.e., tablets) meet the highest quality standards. However, no system is perfect. The European Medicines Agency (EMEA) state that somewhere between 5-10\% of pharmaceutical production batches must be either reworked or discarded, because they do not fully meet the stringent final quality specifications. Poor production quality, often due to inflexible manufacturing and insufficient process understanding represents an unnecessary burden in both time and expense. According to Cedar Management Consulting, the bulk of the global top 16 pharmaceutical companies' budgets are spent on manufacturing $(\sim 36 \%)$, whereas the research and development (R\&D) expenses (often considered to be the major cost burden) can be less than half of this $(\sim 16 \%)$. Therefore, there is great interest in making manufacturing more effective and optimising processes in order to deliver consistent high quality.

\section{Research objective}

The goal of this research is to facilitate greater process/production understanding and knowledge through the systematic evaluation of the manufacture of a modified-release (MR) oral tablet. The active ingredient in the MR tablet investigated in this research, along with many of the more recently developed drugs, exhibits low solubility, which provides additional challenges for consistent drug release and absorption. Pharmaceutical oral tablets remain the most popular drug delivery mechanism. MR tablets are principally used when the duration of action of a short-acting drug needs to be prolonged. Pharmaceutical companies also reinvent existing drugs by creating modified-release versions, as a legitimate means of patent extension, thus protecting their product and income integrity. The simplest method for modified-release in oral tablets is a matrix delivery system which incorporates hydrophilic polymers (hypromellose) that swells in the presence of water to 
Table 1: Table 1Typical ingredients used in a modified-release tablet, showing the $\%$ composition and function

\begin{tabular}{|l|c|c|}
\hline \multicolumn{1}{|c|}{$\begin{array}{c}\text { Typical IngredientsIn a MR } \\
\text { Tablet }\end{array}$} & TypicalComposifionction of Materials (API \& Excipients) \\
\hline $\begin{array}{l}\text { Active Pharmaceutical Ingredi- } \\
\text { ent (API) }\end{array}$ & $\sim 18 \%$ & Active Medicine (Drug Ingredient) \\
\hline $\begin{array}{l}\text { Calcium hydrogen phosphate } \\
\text { dihydrate }\end{array}$ & $\sim 53 \%$ & Diluent(To bulk up the tablet) \\
\hline Hypromellose (100 \& $4000 \mathrm{cP})$ & $\sim 21 \%$ & Matrix agent(To control drug release) \\
\hline Magnesium stearate & $\sim 0.05 \%$ & $\begin{array}{c}\text { Lubricant(To ensure that tablet ejects } \\
\text { efficiently from the tablet press) }\end{array}$ \\
\hline Maltodextrin & $\sim 7 \%$ & $\begin{array}{c}\text { Binder(To hold the different ingredients } \\
\text { together) }\end{array}$ \\
\hline Silica, colloidal anhydrous & $\sim 0.2 \%$ & $\begin{array}{c}\text { Flow agent(To ensure good powder and } \\
\text { granule flowability) }\end{array}$ \\
\hline Water & $<1 \%$ & \begin{tabular}{c} 
Granulation liquid \\
\hline
\end{tabular}
\end{tabular}

form a gel layer around the tablet to control the release of the active components from the tablet.

\section{Pharmaceutical manufacturing}

Pharmaceutical processes use batch recipes which are established during R\&D and subsequently scaled up for full production. Product development combines the active drug with a variety of other ingredients, Table 1 shows some typical ingredients used in the manufacture of a MR tablet. The full scale batch manufacturing regime is fixed early in the lifecycle of the product, following the process validation of three production runs generating an acceptable (though perhaps not optimal!) product quality.

The pharmaceutical industry defines validation as the documented act of demonstrating that procedures, processes, and production activity will consistently lead to the expected results. Validation includes the qualification of systems and equipment and is a requirement for Good Manufacturing Practices (GMP) and other regulatory controls. Modifiedrelease tablets containing poor solubility drugs are particularly susceptible to process variability; dissolution is a critical quality attribute for tablets. Figure 1 illustrates how observed dissolutions can deviate from a normal distribution.

Previous studies into the production of oral tablet drugs have shown that a large number of factors including the physicochemical (physical \& chemical) properties of the raw materials and the manufacturing processes used, can influence drug release behaviour from the final product i.e., dissolution. More recently, the focus of regulatory bodies such 


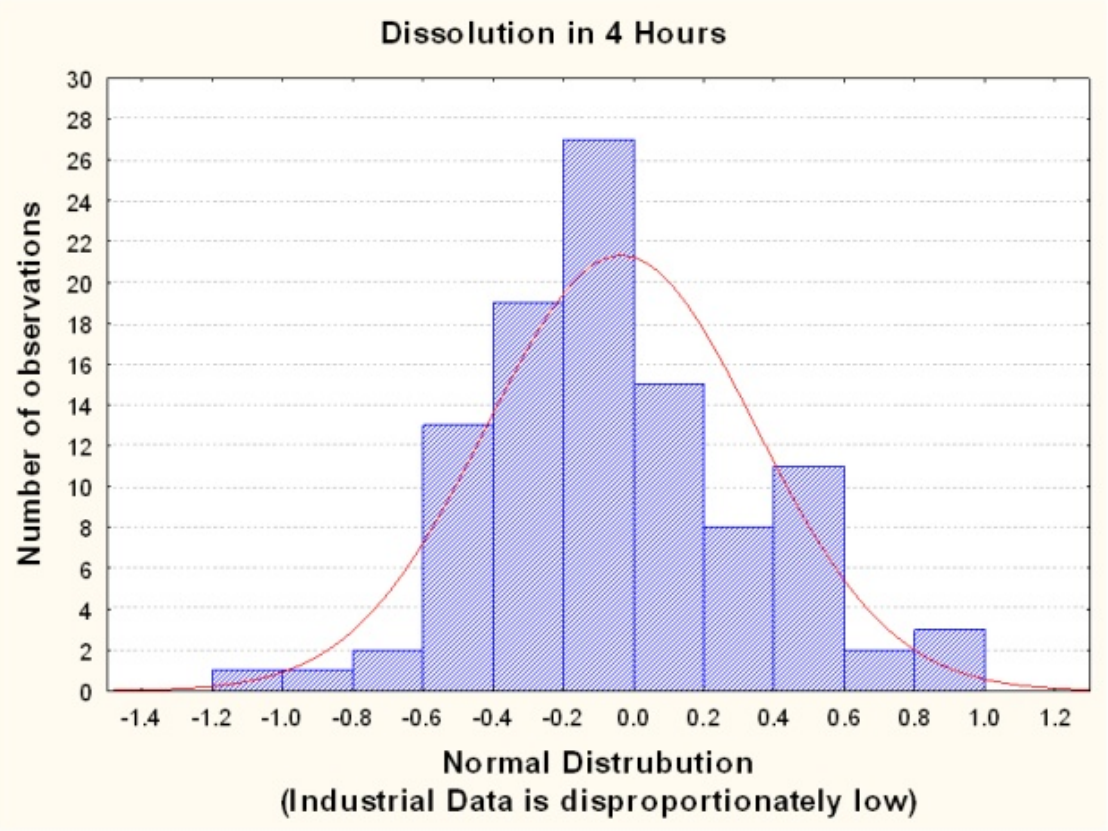

Figure 1: The industrial data analysed showed how real measured dissolution values (the bars) deviated from normal distribution (the line) expected by commercial specification.

as the Irish Medicines Board (IMB) and the U.S. Food and Drugs Authority (FDA) on operating validated inflexible processes has shifted. Pharmaceutical companies are now required to demonstrate an in-depth understanding of the dynamic behaviour of ingredients/production and the impact of this on the finished product performance.

Quality by Design (QbD) is the mechanism used to provide an increased understanding and assurance of product quality. $\mathrm{QbD}$ is a systematic concept to product development as opposed to the traditional, more empirical method. Under the QbD framework, Process Analytical Technology (PAT) employs technology to understand and control the manufacturing process.

\section{Methods of research}

The research was divided into four distinct phases, as seen in Figure 2, with the optimum dissolution specification being the ultimate quality goal.

\section{Industrial data}

Industrial data from the production of a commercial MR tablet was compiled using 105 manufacturing batch records. Analysis of real industrial data was valuable as it indicated the actual quality situation pertaining to production. However, it was also constrained, as many factors recorded in a controlled process do not possess sufficient inherent variability 


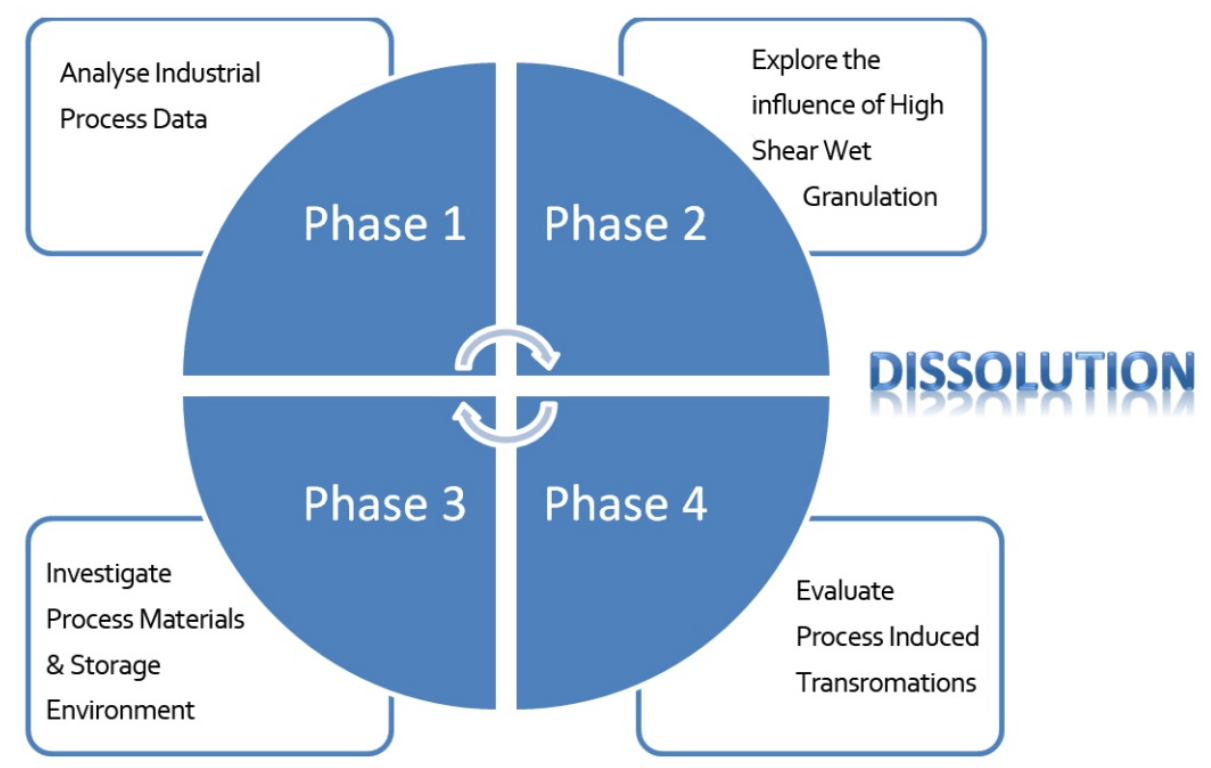

Figure 2: Research phases

to be useful. A coefficient of variance greater than $10 \%$ was used as the acceptance criteria to indicate useful data. I investigated the effect of various process factors using a Pareto analysis (a statistical technique which prioritises effects) using software (Statistica 7.0). I was able to correlate process input factor changes to improved dissolution using Microsoft Excel.

\section{High shear wet granulation}

Granulation is a size enlargement (powder) process designed to assist with drug uniformity, enhance powder flowability and reduce dustiness. High shear wet granulation combines the ingredients of the drug formulation using a high speed mixing blade and chopper, a liquid binder is used to agglomerate the powder particles into granules. This technique is common in the pharmaceutical industry due to its ability to reduce processing time and produce granules with high strength and density. I examined the relationship between the high shear wet granulation operation and products formed to facilitate greater process understanding, thus expediting the application of QbD and PAT to the tableting process. I used a special (L9 Taguchi) structured design of experiments (DOE) which was excellent at minimising the number of experiments needed to be able to reliably predict whether certain processing parameters are better than others. I used the original industrial data as a guide to select various energy inputs (mixing / milling) and material changes (wetting) for analysis. 


\section{Process materials and storage environment}

Quality by design $(\mathrm{QbD})$ relies on process understanding and therefore acquiring information regarding the processes applied, material used or general environment is vital. The QbD approach to product development requires a systematic evaluation and understanding of the formulation and manufacturing process. In this phase of the research a range of different studies were carried out to assess the influence of a variety of process parameters and critical quality attributes of materials on the target product performance of controlled release tablets, i.e., the drug dissolution.

I studied the impact of varying the granulation endpoint criteria, the influence of granule particle size along with excipient (raw ingredients) variability; batch to batch and supplier to supplier. I also investigated the influence of temperature, time and relative humidity during the storage of granules prior to blending and eventual tableting.

\section{Process Induced Transformations (PITs)}

The thermal and mechanical processes involved in the production of pharmaceutical tablets can be very vigorous, involving heating, high shear mixing, wetting, milling, blending, storage and finally compression to form tablets. It is possible that process stresses may cause changes in the materials involved. The general term for these changes is process induced transformations (PITs)

Many drug substances can exist in different crystal arrangements. This phenomenon is called polymorphism. Different polymorphs of the same pharmaceutical solid can have different chemical and physical properties which can alter apparent solubility and the dissolution rate. Solvent molecules can also be incorporated in the crystal lattice called solvates or pseudopolymorphs. If the solvent is water, the polymorph formed is called a hydrate. My research work focused on hydrates, as water was the solvent used in the high shear wet granulation process. I investigated PITs using a range of instrumentation, such as Scanning Differential Calorimetry (DSC) Scanning Electron Microscopy (SEM), Hot Stage Microscopy.

\section{Research findings}

The industrial data analysed indicated the presence of product variability and the importance of the high shear wet granulation step in the overall tablet manufacturing process. Using experimentation, I developed an understanding of how the granulation process affects intermediate materials. Figure 4 illustrates the various significant process interactions identified. 


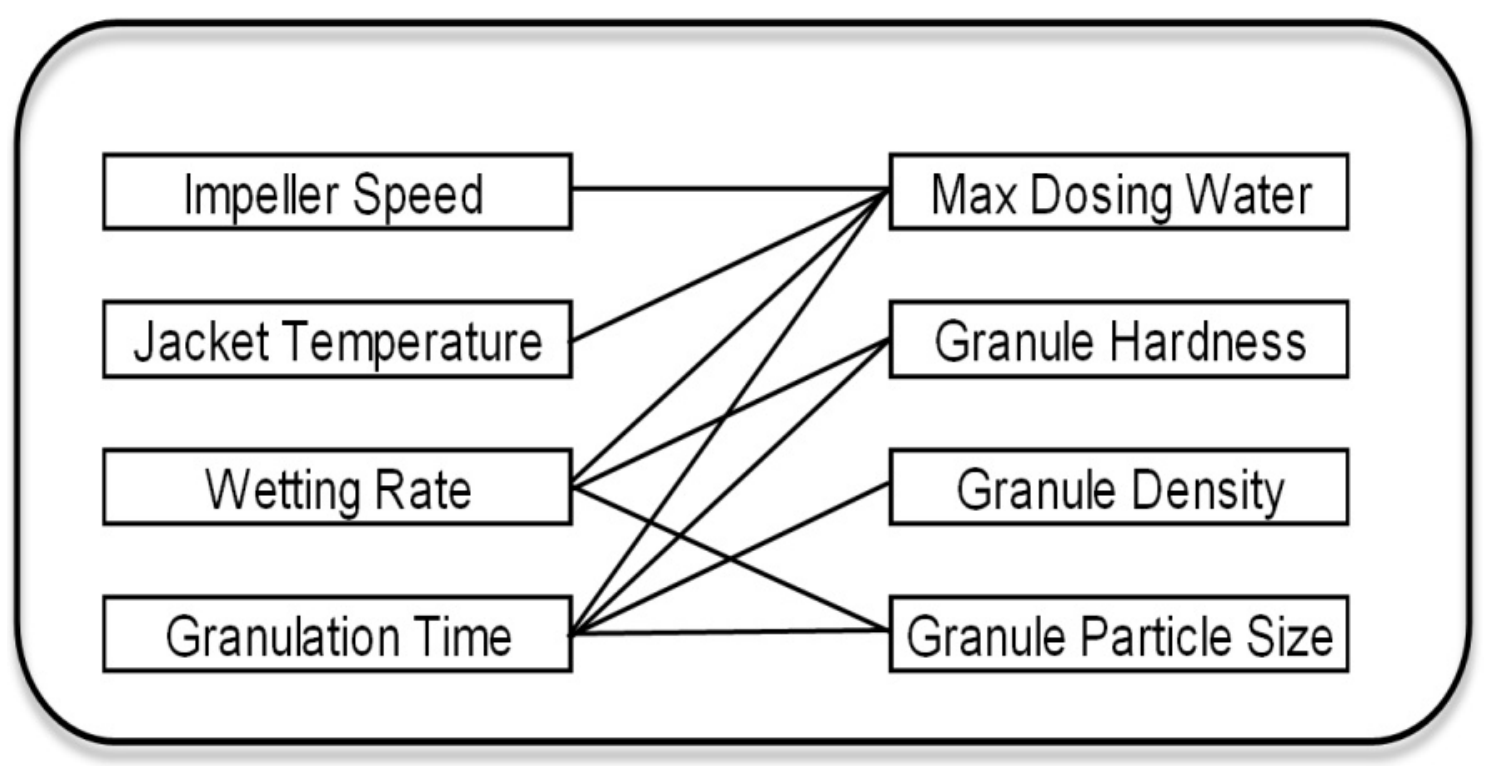

Figure 3: How Process parameters influence the intermediate (granules) material produced

I determined that changes both in supplier and material batch used; resulted in dissolution variability. The same United States Pharmacopeia (USP) grade of hypromellose (a critical component in the controlled release of the drug substance) was used in all cases but different dissolution release profiles were observed shown in Figure 4.

I analysed Intermediate products such as granules using a range of sophisticated techniques to identify if any PITs had occurred. A DSC analysis was carried out on nine granu-

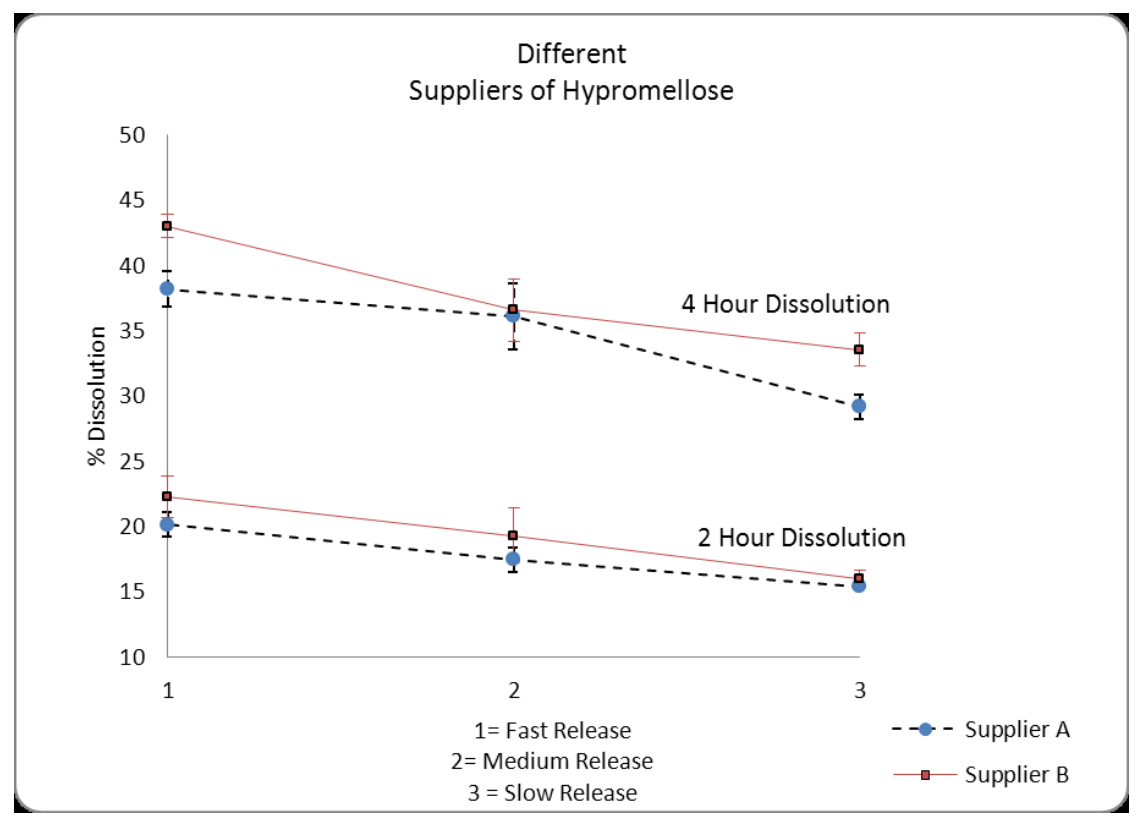

Figure 4: Changes in dissolution were observed when the manufacturer (A/B) of the same raw ingredient was changed 
lations containing the same ingredients but processed using different granulator settings. The DSC measured the amount of heat required to increase the temperature of a sample as a function of temperature. The scans were not identical; some samples indicated that the intense processing regime used for the high shear wet granulation may have produced hydrates under certain conditions. The presences of hydrates can influence subsequent dissolution profiles.

\section{Conclusion}

This research comprehensively examined the challenges faced in the pharmaceutical production of a MR tablet containing a low solubility active ingredient. Quality issues related to drug manufacturing continues to be highly relevant, in 2010 the U.S. Food and Drug Administration reported more than 1,742 drug recalls, skyrocketing from 426 in 2008.

Utilising industrial data, key process variables and materials properties which can influence quality were identified. Laboratory testing based on the industrial data results built up a more comprehensive understanding of the overall production. Dissolution release profiles were modelled and the consistency of batch production techniques was examined.

This work contributes towards the goal of increased process understanding and thus facilitates the integration of quality by design as a process/product optimisation technique. The research is of relevance to anyone involved in the production of medicines, in particular tablets. According to the Irish Pharmaceutical Healthcare Association (IPHA), Ireland currently manufactures 5 of the world's top 12 medicines and is the second largest net exporter of medicines in the world.

I wish to acknowledge help and support of all the staff of the Department of Process \& Chemical Engineering. I would like to especially thank my supervisors, Dr. Jorge Oliveira \& Dr. Abina Crean (School of Pharmacy) 